\title{
An Ab-initio study on UAl for nuclear applications
}

\author{
Yasemin Ö. Ciftci ${ }^{*}$, Aynur Özcan, Irem Ö. Alp \\ Gazi University, Department of Physics, Ankara, TURKEY
}

\begin{abstract}
The structural, elastic, electronic and bonding nature and vibrational properties of UA1 in B2 structure under zero pressure have been investigated by performing first principles calculations using density functional theory. The exchange-correlation potentials were treated within the generalized gradient approximation (GGA). The calculated quantities are agreed well with the available results. The electronic properties such as band structure and density of states reveal that UAl is metallic in nature with large overlap at Fermi level. The single-crystal elastic stiffness constants of UAl are investigated using stress-strain method. Present results for elastic constants show that this compound is mechanically metastable which is agreement with previous study [3]. The chemical bonding is interpreted by calculating the density of states and electron density distribution analysis. UAl has metallic bonding characteristic. The presented phonon dispersion curves and one-phonon DOS is also confirmed that this compound is dynamically unstable.
\end{abstract}

\section{Introduction}

Dispersion nuclear fuels are being developed as fuels for research reactors, which are consists of U-Mo particles dispersed in an Al matrix. The product of the reaction UAlx system has become the subject of numerous studies since the middle of last century owning to its outstanding role in nuclear fuel development [1,2]. Therefore, in order to fully exploit the product material, it is imperative to obtain a good understanding of the physical properties of the UAlx system, and in particular, their structures and dynamic properties. A detailed theoretical study of structural, electronic, and elastic properties of cubic UAlx $(x=1,2,3)$ have been presented employing the pseudopotential plane-wave method based on densityfunctional theory [3].

In this work, we have been discussed the structural, elastic, electronic and vibrational properties of the uranium-aluminium intermetallic $\mathrm{UAl}$ in $\mathrm{CsCl}$ crystal structure by analysing first principles calculations. In this study, lattice parameter is quite convenient with literature and the electronic band structures are determined with density of states and this compound exhibits metallic in the nature. Additionally, the structure is dynamically stable considerable as vibrational part of the study.

\section{Calculation method}

All results have been reported here calculated using Vienna Ab-initio Simulation Package (VASP) which implements Density Functional Theory (DFT) [4-6]. The plane wave based pseudo-potential method has been used for the total energy calculations. The electron-ion interaction and the exchange correlation energy are described under the Projector Augmented Wave (PAW) method and the generalized gradient approximation (GGA) of Perdew Burke Ernzerhof (PBE) scheme, respectively [7-9]. All-electronic projector augmented wave potentials are employed for the elements $\mathrm{U}$ and Al. The cut off energy for the plane wave basis set is fixed at $600 \mathrm{eV}$. For all the structures both the lattice parameters and the atomic coordinates have been relaxed ensuring that energies converge to a precision of less than $10^{-10} \mathrm{eV} /$ atom and Hellmann-Feynman forces to less than $10^{-6} \mathrm{eV} / \AA$. The k-point meshes are constructed using the Monkhorst-Pack scheme and the 14x14x14 k-point mesh is used for the primitive cell for Brillouin zone sampling [10].

\section{Results and discussions}

\subsection{Structural and elastic properties}

The crystal structure of UAl alloy is shown in Fig. 1 in which the Uranium atoms are grey colour and aluminium atom is red one in the middle of the unit cell. The geometry optimization was carried out as a function of the normal stress by minimizing the total energy of the system.

The crystal structure is fully relaxed under a given set of exchange-correlation potential functions and found an equilibrium structure with a minimum total energy. Our calculated value of the lattice constant with GGA-PBE method is obtained as $3.47 \AA$. and is quite compatible with theoretical value [3] (3.51 $\AA$ ). The resulting energy versus volume curve is indicated in Figure 2 . The equilibrium value of the volume is nearly $280 \mathrm{Bohr}^{3}$ at the minimum energy value. 


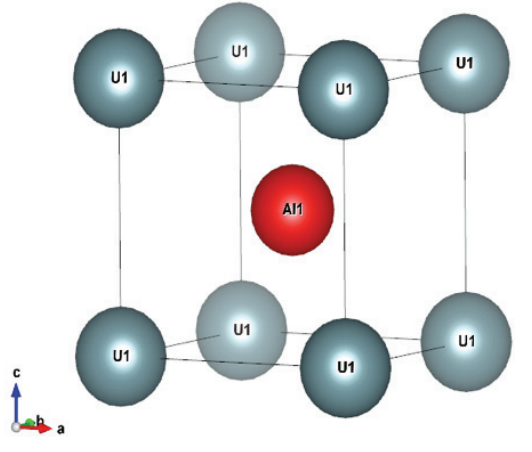

Fig. 1. The crystal structure of UA1 alloy in B2

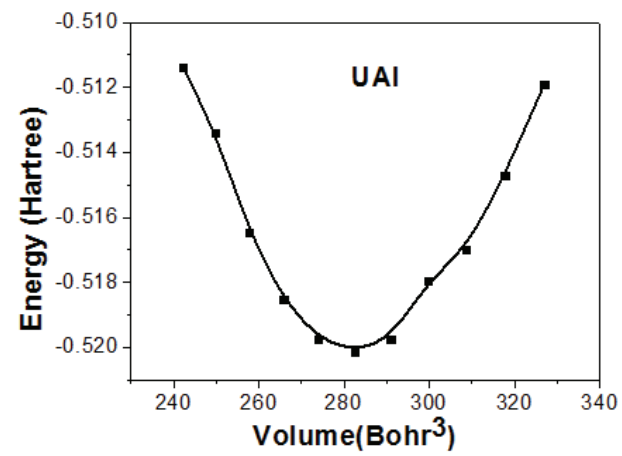

Fig. 2. The energy versus volume graph of UAl crystal structure.

The elastic constants reveal that the response of the crystal to external forces and examine the strength and resistivity of the compounds. For this reason, the second order elastic constants are obtained. To calculate the elastic constants through the first-principles modelling of materials from their known crystal structures, there are basically two common methods [11,12] an approach based on analysis of the total energy of properly strained states of the material in the volume conserving technique and an approach based on the analysis of changes in calculated stress values resulting from changes in the stress-strain technique. In this study, the stress-strain technique is used to determine the second-order elastic constants $\left(C_{i j}\right)$ of UAl alloy in CsCl-type crystal structure.

The Born's stability criteria's [13] should be satisfied for the stability of lattice. The known Born's stability conditions of cubic crystals are: $C_{11}>0, C_{11}-C_{12}>0, C_{44}>0$, $C_{11}+2 C_{12}>0$ and $C_{12}<B<C_{11}$ for mechanical stability. According to the Born's stability conditions, UAl alloy is mechanically unstable using the second order constants for calculations.

Table 1. Calculated equilibrium lattice constant $(a)$, bulk modulus $(B), \mathrm{B} / \mathrm{G}$ ratio, second order elastic constants, Young's modulus $(E)$ and Poisson's ratio of UAl alloy CsCl-type crystal structure* [3]

\begin{tabular}{clccccl}
\hline $\begin{array}{c}a \\
(\AA)\end{array}$ & $\begin{array}{l}B \\
(\mathrm{GPa})\end{array}$ & $B / G$ & $\begin{array}{c}C_{11} \\
(\mathrm{GPa})\end{array}$ & $\begin{array}{c}C_{12} \\
(\mathrm{GPa})\end{array}$ & $\begin{array}{c}C_{44} \\
(\mathrm{GPa})\end{array}$ & $v$ \\
\hline 3.47 & 88.1 & 1.14 & 24.5 & 120.0 & 39.0 & 0.16 \\
$3.51^{*}$ & $82^{*}$ & $-1.73^{*}$ & $22.0^{*}$ & $112.0^{*}$ & $35.0^{*}$ & $-2.85^{*}$ \\
\hline
\end{tabular}

It is known to be such that the bulk modulus $(B)$ determines the resistance of material fracture and the shear modulus $(G)$ represents the resistance to plastic deformation. The $B / G$ ratio is relevant to ductile/brittle behavior for solids. It is well-known that the critical value is $1.75 \mathrm{and} /$ or greater than that value the material is regarded as ductile [14]. UAl alloy indicates that the brittle behaviour due to the fact that the present value of $B / G$ is 1.14 as given in Table 1 at zero pressure.

\subsection{Electronic properties}

Electronic structure calculations are performed to evaluate the electronic properties of UAl alloy in $\mathrm{CsCl}$ type crystal structure along the high symmetry directions in inclusion of total density of states (DOS) with band structure calculated by GGA scheme as presented in Fig. 3.

It can be seen from Fig. 3 that the UAl alloy exhibits the metallic nature of the compound due to the fact that at around Fermi level $\left(E_{F}\right)$ that is set to the zero band structures are nested. According to the band structure and density of states (DOS), there is no band gap. We also have presented the partial density of states (PDOS) and total density of states (TDOS) of UAl alloy for CsCl-type crystal structure in Figure 4.

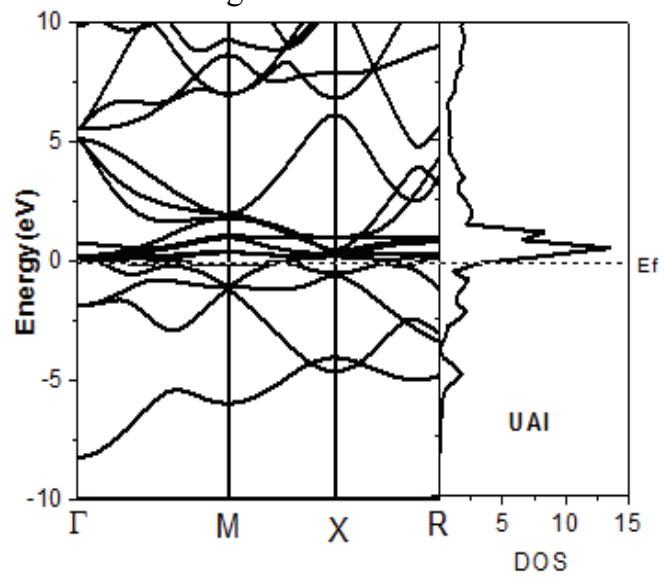

Fig.3. The band structure and density of states of UAl

It is illustrated that in Figure 4, the contribution to the lower valance bands and Fermi energy level are mainly Al-s states and U-d states. The conduction bands are mainly from U- $d$ states and partially Al- $p$ states. The UAl alloy has a metallic behaviour due to the fact that it has no band gap.

\subsection{Vibrational and thermodynamic properties}

The vibrational properties determine the lattice dynamical stability using the phonon spectra of the materials. The phonon frequencies of $\mathrm{UAl}$ alloy in $\mathrm{CsCl}$ structure have been computed using the PHONOPY code $[15,16]$ that is based on the forces obtained from the VASP. The PHONOPY code calculates force constant matrices and phonon frequencies using the density functional perturbation theory as described in Ref. [16]. The present phonon dispersion curves along several high symmetry 
directions using a $2 \times 2 \times 2$ cubic supercell of 16 atoms are illustrated in Figure 5. UAl alloy in $\mathrm{CsCl}$ crystal system has two atoms in the unit cell, in the phonon spectrum has six phonon branches, including three acoustic branches and three optical branches. Therefore, it strongly supports the dynamical unstability of this compound in $\mathrm{CsCl}$ structure.

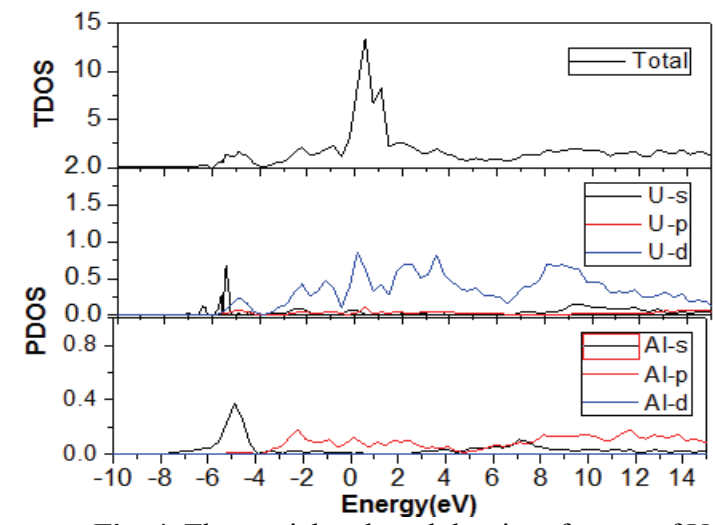

Fig. 4. The partial and total density of states of UAl

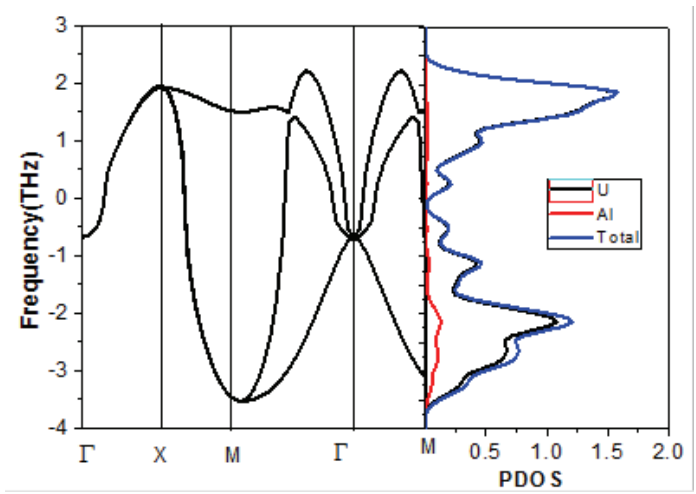

Fig.5. The fonon dispersion curves and phonon DOS of UA1

As shown on the right side of Fig. 5, the phonon density of the states of the UAl alloy consists of two groups of bands. The higher modes with frequencies up to $3.0 \mathrm{THz}$ are composed of the $\mathrm{U}$ atoms. In that region, $\mathrm{U}$ atoms are more dominant than $\mathrm{Al}$ atoms. The modes in the range $-3.5--0.5 \mathrm{THz}$ are predominantly determined by the vibrations of the $\mathrm{U}$ atoms with small contribution $\mathrm{Al}$ atoms.

The temperature dependent properties are also estimated through the thermodynamic quantities based on the quasi-harmonic approach and thermal electronic excitation. By using the phonon frequencies, the thermodynamic parameters such as heat capacity $\left(C_{v}\right)$ and entropy $(S)$ have been determined under quasi-harmonic approximation. The contributions from the lattice vibrations to the entropy and the heat capacity of UAl alloy are also given with Fig. 6. To decrease the probable influence of anharmonicity, the temperature is limited to $1000 \mathrm{~K}$. The contribution from the lattice to the heat capacity follows the Debye model and it approaches Dulong-Petit limit at high temperatures.
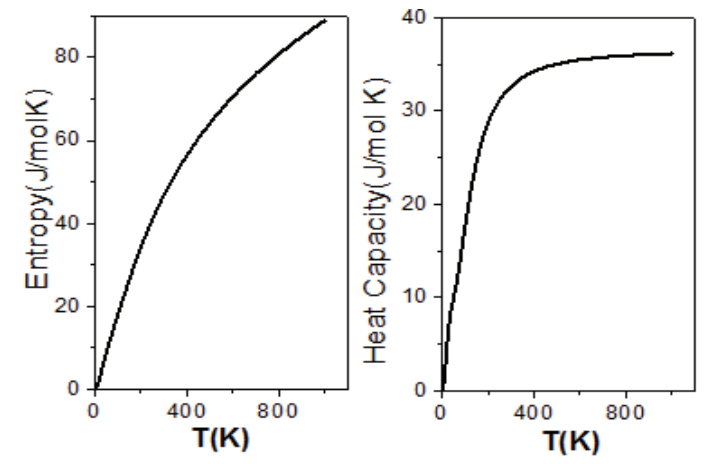

Fig. 6. Phonon thermodynamic properties of UAl

\section{Conclusion}

In conclusion, we have performed the structural, elastic, electronic, vibrational and thermodynamic properties of UAl alloy using first-principles calculations based on the density functional theory with the frame of GGA-PBE. The calculated lattice constant is in good agreement with the existing result from the other related theoretical study. Second-order elastic constants and other mechanical parameters are obtained and mechanical stability for UAl alloy is investigated using the Born's stability criteria. As a result, UAl alloy is determined as mechanically unstable. UAl alloy exhibits a metallic behaviour at zero pressure. Also, vibrational and thermal properties of UAl alloy is investigated and found that UAl alloy is dynamically unstable. Finally, we have deduced that our theoretical results for basic physical properties of UAl alloy that contributes the applications in materials science for bulk systems.

\section{References}

[1] D.D. Keiser Jr, C.R. Clark, M.K. Meyer, Scr. Mater. 51, 893 (2004)

[2] H.J. Ryu et al., J. Nucl. Mater. 321, 210 (2003)

[3] S. Kang, T.Gaon, X. Tian, Physica B 407,748 (2012)

[4] Ning, Y., Gold Bull., 1, 38 (2005)

[5] Golyev, B.B., Synthesis of Alloys. (Metallurgy Press, Moscow, 1984)

[6] Ahmad, S.; Ahmad R.; Jalali-Asabadi S.; Ali Z.; Ahmad I., J. of Magnetism and Magnetic Materials, 422, 458 (2017)

[7] Kresse, G.; Hafner, J., Phys. Rev. B, 49,14251(1994)

[8] Kresse, G.; J. Furthmuller, J., Comput. Mater. Sci. 6 ,15 (1996)

[9] Kohn, W.; Sham, L., Phys. Rev. A , 40,1133 (1965).

[10] Perdew, J.P.; Burke, K.; Ernzerhof, M., Phys. Rev. Lett., 77, 3865 (1996)

[11] Hellman, H., Introduction to Quantum Chemistry. (Leipzig Deuticke, 1937)

[12] Monkhorst, H.J.; and Pack, J.D.; Phys. Rev. B, 13, 5188, (1976)

[13] Chao, C.; Luo, H.L.; Duwez, P., J. Appl. Phys., 34, 7 (1963)

[14] Mehl, J., Phys. Rev. B, 47,2493 (1993)

[15] Bannikov, V.V.; Shein I.R.; Ivanovskii, A.L., Phys. Status Solidi (RRL), 1,89 (2007)

[16] Johnston, I. et al., Solid State Physics Simulations, The Consortium for Upper-Level Physics Software, (John Wiley New York, 1996) 